\title{
An Improved Technique for Image Deblurring
}

\author{
S.Vijayakumar, A. Thilagavathy, Dommaraju Digvijay, Cheekatimarla Abhishek, Bommi \\ Reddy Maneesh Reddy
}

\begin{abstract}
The predicament in image deblurring, that is, the resurgence of an image against the noise along with the blur is an important issue in task dealing with image processing applications. .Owing to the deblurrng issue, many techniques related to regularization has found a concern in the recent years. In this paper, an efficient regularization method is put forward which utilizes the Lagrangian multiplier for the deblurring process. The major contribution of the proposed scheme is to determine the values of regularization approach towards attaining an better enhanced tradeoff among image resurgence and noise restraint using projection based image deblurring.
\end{abstract} multiplier

Keywords : image deblurring,regularization, Lagrangian

\section{INTRODUCTION}

Blurring in images occur owing to a lots of cause such as flaws while capturing the images, very low lighting in the scenario of image capture and other natural causes that exist in the environmental issues. In many image processing applications, the image plays a vital medium of source for further advancements of study pertaining to their related work. But in reality, these images are represented to project the real visual impact of the original image in real world domain. But the captured images are depicted in terms of image medium with low dimensionality. Due to this, certain important information in the image may be hidden or lost in due process. So it becomes necessary to regain the lost information to get the real visual impact of the image along with intact information the image contains within it. The blurring types are average blur, Gaussian blur and motion blur. A blurred image is usually represented as $B=d * I+n$ where $\mathrm{B}$ is the blurred image, $\mathrm{d}$ is the distortion operator ,

Revised Manuscript Received on December 30, 2019.

* Correspondence Author

S.Vijayakumar*, Associate Professor, Department of CSE, R.M.K Engineering College, Kavaraipettai, Tamil Nadu, India. Email: svk.cse@rmkec.ac.in

Dr.A.Thilagavathy, Associate Professor, Department of CSE, R.M.K Engineering College, Kavaraipettai, Tamil Nadu, India. Email: atv.cse@rmkec.ac.in

Dommaraju Digvijay, Student, IV Year CSE, Department of CSE, R.M.K Engineering College, Kavaraipettai, Tamil Nadu, India. Email: gotan.digvijay@gmail.com

Cheekatimarla Abhishek, Student, IV Year CSE, Department of CSE, R.M.K Engineering College, Kavaraipettai, Tamil Nadu, India. Email: abhishek.chikatimarla@gmail.com

Bommi Reddy Maneesh Reddy, Student, IV Year CSE, Department of CSE, R.M.K Engineering College, Kavaraipettai, Tamil Nadu, India. Email: maneeshbommireddy@gmail.com

(C) The Authors. Published by Blue Eyes Intelligence Engineering and Sciences Publication (BEIESP). This is an open access article under the CC BY-NC-ND license (http://creativecommons.org/licenses/by-nc-nd/4.0/) also called as Point Spread Function(PSF), I is the original image and $\mathrm{n}$ is the noise.

Many works has been put forward in recent years for the process of deblurring in images. The main focus depends on getting the original information back from the blurred image. But many of the existing works does not provide accurate information. Much of the existing methods are not very efficient.

A number of algorithms are used for restoration processes that are mainly iterative in nature. In [1], iteration method is used to lower the noise that exists in the image. The iterations are predetermined. Most favorable accuracy [2,3] is obtained in probabilistic models. In [4], optimization strategy is used for restoring the information from noise. Geometric model is used in [5] for deblurring process.

\section{RESEARCH METHOD}

The difficulty of image deblurring, that is to be precise, the resurgence of an image against noise present in the image, have comprehensively considered in the precedent years. Numerous conventional iterative elucidation algorithms continue to exist. Due to the deblurrng issue, many techniques related to regularization has found a concern in the recent years. The techniques use the apriori information to find out a better solution for the blurring problem.

The algorithms make use of the information related to the noise that is determined apriori to give a better solution. The apriority information is also used to find the best possible solution and retaining the useful content hidden in the image due to the presence of noise. In this paper, an efficient regularization method is put forward which utilizes the Lagrangian multiplier for the deblurring process. The major contribution of the proposed scheme is to determine the values of regularization approach towards attaining an better enhanced tradeoff among image resurgence and noise restraint using projection based image deblurring. The major benefit of the planned scheme is that the efficacy is demonstrated experimentally and the predicament in motion deblurring can be solved.

\section{Entry Level Design}

User enters into the selection of image after login. If the user is new one, then register him and then login to the selection of image. After browsing and selecting the image, iterative regularization function is applied to the image. Next step is fine granularity function. Here variational image deblurring is used. After fine granularity, spatial adaptive regularization is functioned on the same image using deterministic annealing. Finally the output image is got from previous functions. User will get the output image.

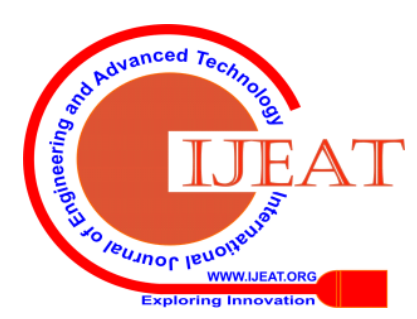




\section{An Improved Technique for Image Deblurring}

\subsection{Iterative regularization}

The projection method utilizes the apriori information that will be used to get the solution. The observation restraint scheme contains the image which is blur. In addition the observation restraint scheme also contains the deprivation model .The

regularization scheme contains the apriori information. The deprivation model can be depicted as in equation (1).

$$
\mathrm{U}_{\mathrm{i}}=\mathrm{OB}_{\mathrm{i}}+\mathrm{g}
$$

where $U_{i}$ is the unblurred image

$\mathrm{B}_{\mathrm{i}}$ is the blurred image

$\mathrm{O}$ is the blurring operator

$\mathrm{g}$ is the Gaussian noise

Further booming progress of image regularization procedures follow the mostly inclined 'disparity principle'. The disparity principle uses the regularization scheme depicted as $F\left(B_{i}\right)$.

The regularization scheme replicates the apriori information regarding $\mathrm{Bi}$. It then applies various calculus operations to attain the projection operator.

The regularization scheme plays an important role in diminishing the blurring level by using the regularization constraint. The constrained optimization problem domain can be converted to an unconstrained optimization problem domain by using the Lagrangian multiplier Ĺ. The bridging amongst the image resurgence and noise restraint is improved by the Lagrangian multiplier L. The choice of regularization constraint determines the effectiveness of the deblurring process. In some applications, filtering heuristics are applied along with regularization constraint to give better efficacy. The major difficulty arises in obtaining the $\mathrm{F}\left(\mathrm{B}_{\mathrm{i}}\right)$ trough analogous projection operator. The main challenge is to minimize the $F\left(B_{i}\right)$. The minimization can be obtained by means of consecutively applying the projection operator.

The extent of regularization is restricted through the Lagrangian multiplier Ĺ. The main drawback in projection based method is determining $\mathrm{F}\left(\mathrm{B}_{\mathrm{i}}\right)$ using the single projection operator. Hence an improved scheme is needed to manage the regularization constraint used in projection based methods. The Lagrangian multiplier $\mathrm{L}$ used in the regularization constraint is scaled down to $1 / \mathrm{n}$ times.

\section{EXPERIMENTAL RESULTS}

Finally the results obtained using above all modules is to be analyzed here and the output images are obtained. This module contains the final output image which is the output of fine granularity and spatial adaptivity. Finally a graph is shown which displays the iteration vs. isnr values in $\mathrm{db}$ for combined results of fine granularity and spatially adaptive regularization which shows the effectiveness of our proposed method compared to iterative regularization.

The purpose of testing is to discover errors. Testing is the process of trying to discover every conceivable fault or weakness in a work product. It provides a way to check the functionality of components, sub assemblies, assemblies and/or a finished product. It is the process of exercising software with the intent of ensuring that the Software system meets its requirements and user expectations and does not fail in an unacceptable manner.

\section{CONCLUSION}

In this paper, an efficient regularization method is put forward which utilizes the Lagrangian multiplier for the deblurring process. The major contribution of the proposed scheme is to determine the values of regularization approach towards attaining a better enhanced tradeoff among image resurgence and noise restraint using projection based image deblurring.

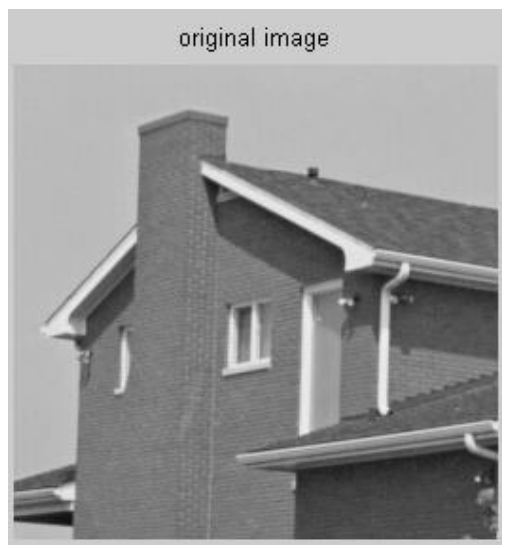

Figure 1: Original Image

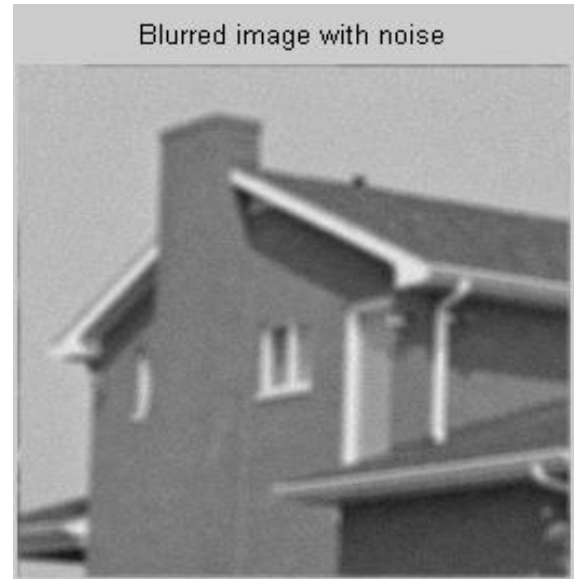

Figure 2: Blurred image with noise

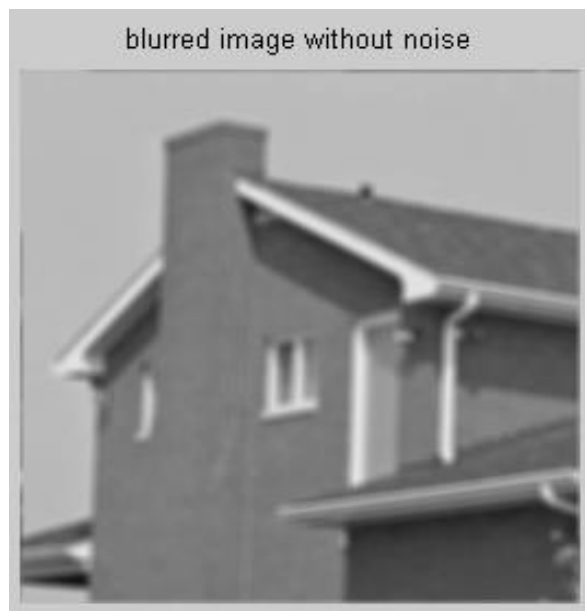

Figure 3: Blurred image without noise 


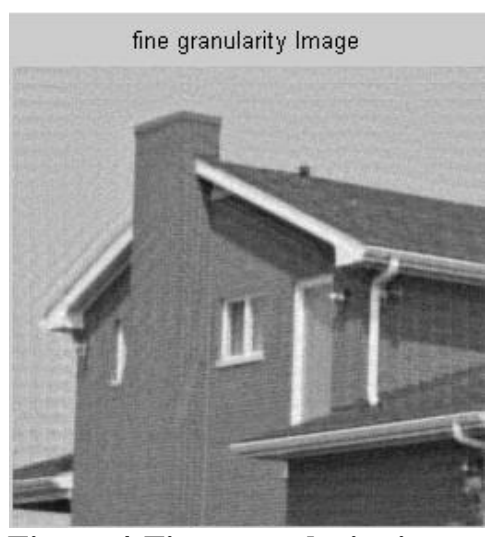

Figure 4:Fine granularity image

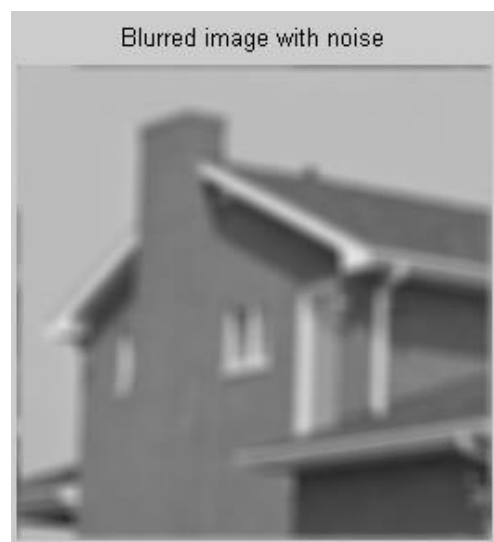

Figure 5 : Blurred Image with noise in Spatial Adaptive Regularization

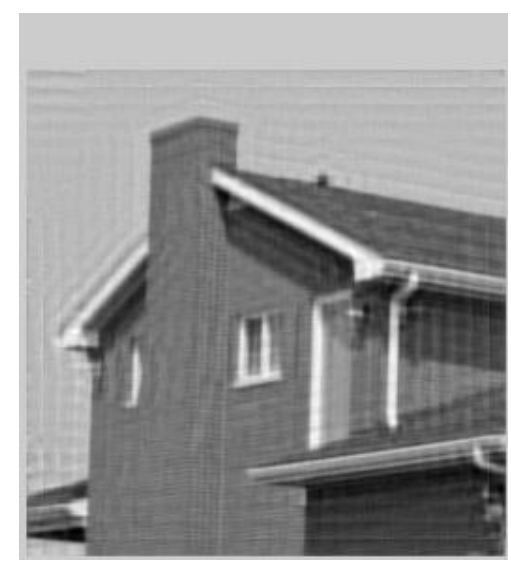

Figure 6 : Blurred Image without noise in Spatial Adaptive Regularization

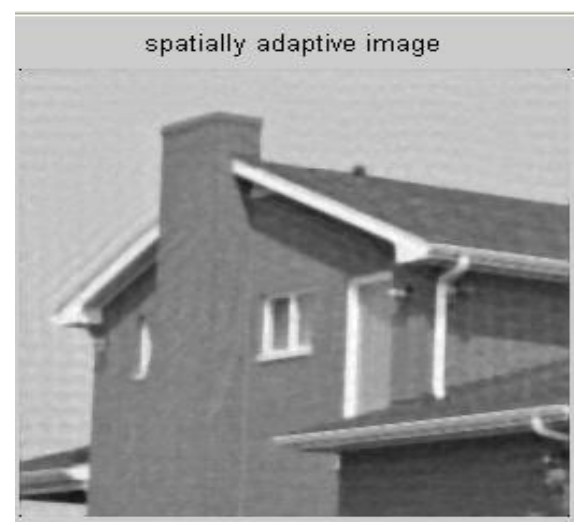

Figure 7: Spatial Adaptive Image
Figure 8: Blurred Image with noise in both Fine Granularity and Spatial Adaptive Regularization

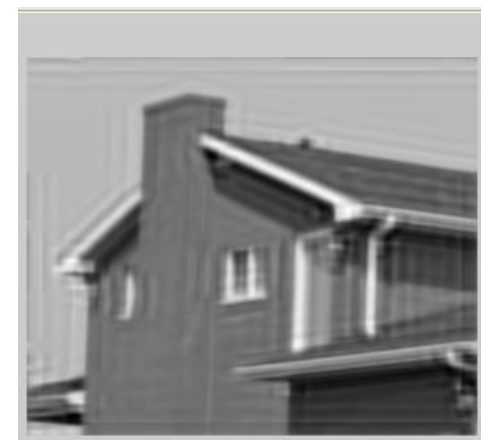

Figure 9: Blurred Image without noise in both Fine Granularity and Spatial Adaptive Regularization

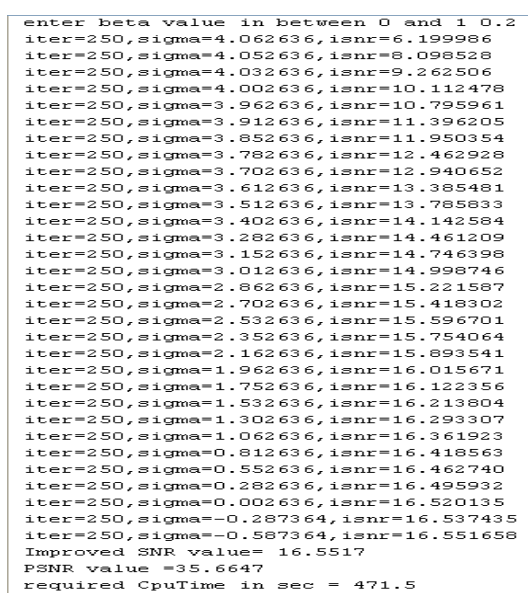

Figure 10: Beta value for process and details about process

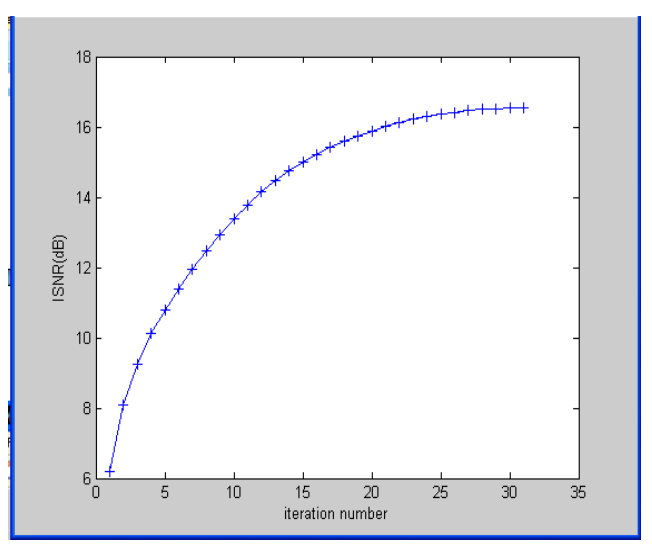

Figure 11 Graph for both Fine Granularity and Spatial Adaptive Regularization 


\section{An Improved Technique for Image Deblurring}

\section{REFERENCES}

1. Biemond, J, Lagendijk, R, and Mersereau.R, 1990, "Iterative methods for image deblurring," Pro. IEEE, vol. 78, no. 5, pp. 856-883.

2. Land weber,L, 1951 "'An iteration formula for Fredholm integral equations of the first kind," American journalof Mathematics , pp. 615-624.

3. Qi Shan, JiayaJia, Aseem Agarwala,"High-quality motion deblurring from a single image", SIGGRAPH, 2008.

4. Jian-Feng Cai, Hui Ji, Chaoqiang Liu, Zuowei Shen, Blind motion deblurring from a single image using sparse approximation", CVPR, 2009.

5. O, Whyte, J, Sivic, A, Zisserman, J,Ponce,"Nonuniformed blurring for shaken images", in Proceedings of the IEEE Conference on Computer Vision and Pattern Recognition, 2010.

\section{AUTHORS PROFILE}

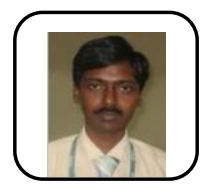

S. Vijayakumar received his B.E. (Computer Science and Engineering) from University of Madras, M.E. (Computer Science and Engineering) from Anna University and pursuing Ph.D in Anna University. He is working as Associate Professor in Computer Science College. Area of interest is Image Processing.

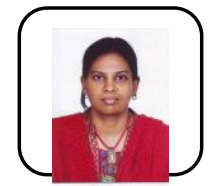

Dr.A. Thilagavathy received her B.E. (Computer Science and Engineering) from University of Madras, M.E. (Computer Science and Engineering) from Anna University and Ph.D from Anna University. She is working as Associate Professor in Computer Science and Engineering department at R.M.K. Engineering College. Area of interest is Image Processing and soft computing

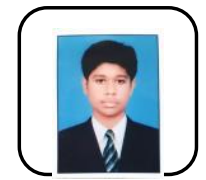

Dommaraju Digvijay, is fourth year student of Computer Science and Engineering, R.M.K. Engineering College

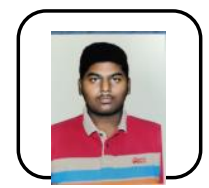

Cheekatimarla Abhishek, is fourth year student of Computer Science and Engineering, R.M.K. Engineering College

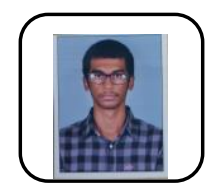

Bommi Reddy Maneesh Reddy, is fourth year student of Computer Science and Engineering, R.M.K. Engineering College 